\title{
Análise do engajamento no contexto da formação de professores de Música utilizando a Openredu
}

\author{
Manassés Bispo da Silva ${ }^{1}$, Alex Sandro Gomes ${ }^{1}$ \\ ${ }^{1}$ Centro de Informática - Universidade Federal de Pernambuco (UFPE) \\ Caixa Postal 7851 - 50732-970 - Recife - PE - Brazil \\ $\{$ mbs 4, asg $\} @$ cin.ufpe.br
}

\begin{abstract}
This article summarizes a master's work in Computer Science, evaluating the effectiveness of mixed mode with the use of educational social network on student engagement in learning music. The research is experimental and almost happened to graduating students of a Degree in Music. Data collection was triangularizada with observation and questionnaire online application and semistructured interviews. The results point to student engagement evidence, and consequently improving the quality of education, from the discussion of factors that positively or negatively impact the engagement of students in the light of Activity Theory.
\end{abstract}

Resumo. Este artigo sintetiza um trabalho de mestrado em Ciência da Computação, avaliando a efetividade da modalidade mista com o uso de rede social educativa sobre o engajamento discente na aprendizagem musical. A pesquisa é quase experimental e ocorreu com alunos concluintes de um curso de Licenciatura em Música. A coleta de dados foi triangularizada com observação e aplicação de questionário online e entrevista semiestruturada. Os resultados apontam para indícios de engajamento discente, e consequente melhoria da qualidade do ensino, a partir da discussão sobre fatores que podem impactar positivamente ou negativamente o engajamento dos alunos, à luz da Teoria da Atividade.

\section{Introdução}

As constantes mudanças que ocorrem nos processos de ensino e aprendizagem instigam uma melhoria contínua na interação de informações. Paralelamente, a globalização e a necessidade de racionalização do tempo exigem o desenvolvimento de novos métodos de ensinar e de aprender, pelo que a inovação tecnológica tem assumido um papel vital na educação [Valdez, Ferreira e Barbosa 2013], favorecendo a aprendizagem colaborativa [Rodrigues et al. 2011].

Utilizada amplamente em diferentes áreas do conhecimento, a educação a distância (EAD) consiste no processo de ensino e aprendizagem sem interação face a face, sendo a construção do conhecimento mediada pela tecnologia, a partir da comunicação interativa entre os usuários. Este trabalho se insere numa modalidade que combina interações em EAD com encontros presenciais, modalidade esta conhecida como mista ou semipresencial (do inglês blended learning). 
Embora a procura por cursos de Música a distância no Brasil seja crescente [Oliveira-Torres 2012; Gohn 2011; Henderson Filho 2007], estudos defendem que de um modo geral os alunos prefeririam a modalidade mista à modalidade estritamente a distância, justamente pela valorização das interações presenciais, facultadas por aquela modalidade [Sheridan 2009; Schneider et al. 2013]. É importante salientar que, desde séculos, a interação presencial entre aluno e professor é fundamental no ensino da performance musical, dada a complexidade inerente a este campo de ensino. E justamente por ser complexo, o ensino da prática de instrumentos a distância ainda é bastante desafiador. Entretanto, o ensino de disciplinas mais teóricas seria totalmente compatível com as modalidades a distância ou mista [Gohn 2011].

Dentre os 7 cursos de nível superior em Música na modalidade a distância constantes no portal do Mistério da Educação (<http://www.mec.gov.br/>), nenhum deles utiliza uma rede social educativa senão, em sua maioria, o Moodle, que é uma plataforma consolidada internacionalmente no meio acadêmico. Outrossim, não foram identificadas pesquisas publicadas que indicassem o uso da modalidade mista com redes sociais educativas no âmbito do ensino de Música, tornando emergente o tema desta pesquisa no Brasil. Inclusive, em detrimento das plataformas tradicionais, as redes sociais educativas podem ter maior aceitação por parte dos discentes, já que estão comumente apropriados das redes sociais [Rosa 2012 e Soares 2012].

A democratização do ensino de Música na modalidade mista e com redes sociais educativas poderá atender de forma eficiente e agradável à crescente demanda da Lei 11.769 [Brasil 2008], a qual garante o ensino de Música nas escolas da educação básica. Também poderá contribuir para a melhoria da qualidade do ensino nas escolas específicas de Música e, ainda, para a formação docente em Música, que segundo Colabardini e Oliveira (2013) tem de ser ampliada. A utilização de uma rede social educativa, mesmo como ferramenta auxiliar na modalidade presencial, poderá servir à qualidade do ensino e da aprendizagem musical, inclusive na educação básica.

Deste modo, o objetivo principal do trabalho é avaliar a efetividade da modalidade mista com o uso de uma rede social educativa sobre o engajamento discente na aprendizagem musical. Os objetivos específicos compreendem a identificação de fatores que promovem ou prejudicam o engajamento dos alunos, bem como a descrição das relações entre o uso da modalidade mista e o engajamento discente, com o uso da rede social educativa Openredu.

\section{Engajamento na modalidade mista}

O conceito de engajamento está relacionado à predisposição do indivíduo em participar de uma atividade [Campello 2005]. Concerne à retenção e motivação dos alunos, e o consequente sucesso institucional [Yonezawa, Jones e Joselowsky 2009]. Logo, a ideia de aluno engajado remete à concepção de qualidade do processo de ensino e aprendizagem [Kuh 2001]. Para Tang (2010), a abertura ao ensino interativo promove a iniciativa de aprendizagem e capacidade de exploração dos alunos. Neste modelo de aprendizagem mista, não só o professor pode desempenhar o papel principal, como no ensino tradicional, mas também os alunos podem desempenhar o papel principal, como agentes ativos no processo. Os estudos de Tang (2010) mostram que este modelo pode melhorar o pensamento e a capacidade de inovação dos estudantes. 
Em detrimento do suposto isolamento causado pelo computador, Sylvestre (2012), que examina as práticas sociais que permeiam as redes sociais, propõe o uso de redes sociais na aprendizagem, salientando a relevância das interações digitais e a formação crítica que a escola pode oferecer aos alunos, a partir da adoção dessas tecnologias. Embora Yang (2003) afirme que o uso de redes sociais não seja determinante no desempenho dos alunos, Morais et al. (2011) e Sylvestre (2012) atestam a potencialidade das redes sociais na aprendizagem. Diante disso, Ambiente Virtual de Aprendizagem - $\mathrm{AVA}^{1}$ configurado como uma rede social seria a tecnologia mais convincente para o sucesso do processo educativo, visto que aos sujeitos estaria facultada maior possibilidade de interação.

\subsection{As redes sociais educativas}

A potencialidade das redes sociais está nas práticas de interação e formação de grupos de interesse que estão na origem das narrativas digitais da sociedade do conhecimento. O sentido da construção coletiva e colaborativa na Web constitui uma das principais características destas organizações, para além da flexibilidade e da complexidade dos sistemas de informação, aprendizagem e conhecimento [Morais et al. 2011]. Uma rede social é dita educativa quando é concebida para propósitos de ensino e aprendizagem. As redes sociais (especialmente as educacionais) buscam fomentar um novo paradigma de AVAs, em que professores, alunos e conteúdos convivem, interagem e se aproximam, sem barreiras, sem burocracias [Gomes et al. 2012].

As redes sociais educacionais mais conhecidas são $E d m o d o^{2}$, teamie $^{3}$, tria $^{4}$ (das editoras Ática e Scipione), e Openredu ${ }^{5}$, sendo estas duas últimas nacionais. Oliveira e Oliveira (2012) citam a Schoology ${ }^{6}$, a Lore $^{7}$, a Passei direto ${ }^{8}$ e a Ebah $^{9}$, sendo estas três últimas voltadas para o público do ensino superior. Há também a Students Circle Network $^{10}$, a germânica StudiVZ ${ }^{11}$, a polonesa Nasza-klasa.pl ${ }^{12}$, a Classmates.com ${ }^{13}$ e a Academia.edu ${ }^{14}$, muito difundida no Brasil entre acadêmicos e pesquisadores. A rede social educativa utilizada nesta pesquisa foi a Openredu, um AVA cujas características foram desenvolvidas com intuito de otimizar a prática de ensino-aprendizagem e gerir novas possibilidades de ensino, acessíveis por meio de interfaces de acesso simplificado e intuitivo [Gomes et al. 2012]. Foi escolhida para esta pesquisa não somente pelo acesso ao suporte, já que fora concebida pela UFPE e por ter seu uso respaldo por várias pesquisas, como aponta Gomes et al. (2012).

\footnotetext{
1 Também conhecidos como LMS, do inglês Learning Management System.

2 Disponível em: < http://www.edmodo.com/?language=pt-br >.

3 Disponível em: < http://theteamie.com/>.

4 Disponível em: < http://www.redetria.com.br/_layouts/Paginas/niciais/Inicio.aspx >.

5 Desenvolvida pelo Grupo de Pesquisa em Ciências Cognitivas e Tecnologia Educacional CCTE do Centro de Informática da UFPE. Disponível em: <openredu.cin.ufpe.br>.

6 Disponível em: < https://www.schoology.com/home.php >.

7 Disponível em: < http://lore.com/ >.

8 Disponível em: < http://passeidireto.com/ >.

9 Disponível em: < http://www.ebah.com.br/ >.

10 Disponível em: < http://studentscircle.net/about/us>.

11 Disponível em: < http://www.studivz.net/Default >.

12 Disponível em: < http://nk.pl/ >.

13 Disponível em: < http://www.classmates.com/ >.

14 Disponível em:< http://academia.edu/ >.
} 


\subsection{Engajamento no contexto da modalidade mista}

O ensino a distância faculta que a mensuração tradicional de envolvimento dos alunos, a exemplo da frequência em sala de aula, seja substituída por outras formas de avaliação de engajamento, através de uma gama de dados registrados pelo AVA utilizado.

A ideia de aluno engajado remete à concepção de qualidade do processo de ensino e aprendizagem [Kuh 2001]. Os AVAs estão sendo adotados por muitas universidades, e servem como meio de registro e acompanhamento do comportamento de alunos, nas modalidades EAD, mista e mesmo na modalidade presencial, em que AVAs sejam utilizados como ferramentas auxiliares, conforme tendência apontada pela literatura [Beer, Clark e Jones 2010]. O estudo destes autores, por exemplo, mostra que a adoção da plataforma Moodle na CQUniversity gerou padrões nos dados, os quais podem ser usados como indicadores de engajamento discente.

\section{Referencial teórico}

A Teoria da Atividade (TA) tem origem na Psicologia, no início do século XX, com Vygotsky e seus alunos Leontiev e Lúria, e trata a atividade humana como o ponto central para o entendimento da formação dos processos mentais humanos, buscando uma compreensão mais esclarecedora sobre nosso comportamento.

No âmbito da Computação, o interesse sobre a TA surgiu inicialmente na comunidade de Interação Humano Computador, que vem discutindo o potencial dessa teoria como uma estrutura de fundamentação para pesquisas e projetos [Lim e Hang 2003; Bertelsen e Bødker 2003]. Mais recentemente, a comunidade de Engenharia de Requisitos, começa explorar possibilidades de uso da Teoria da Atividade para a captura, modelagem e definição de requisitos de software [McGrath e Uden 2000]. Citados por Barbosa e Silva (2010), Bertelsen e Bødker (2003) listam os seguintes pontos em que a Teoria da Atividade se concentra: análise e design de uma prática específica e contextual; análise e design com foco no uso real e na complexidade da atividade multiusuário e, em particular, na noção essencial do artefato como mediador da atividade humana; o desenvolvimento da experiência e do uso em geral; e, finalmente, a participação ativa do usuário no design, e com foco no uso como parte do design. Portanto, nesta pesquisa a TA possibilitou a análise tanto do engajamento dos usuários quanto da usabilidade da Openredu.

\section{Procedimentos metodológicos}

A pesquisa teve uma abordagem qualitativa [Lakatos e Marconi 1991]. A fim de proceder com a triangularização dos dados, a coleta de dados contou com a captura de tela, com a aplicação de questionário, distribuído conforme as recomendações exaradas por Barbosa e Silva (2010), observação do AVA e entrevistas semiestruturadas, conforme Lakatos e Marconi (1991) e Malheiros (2011). Essas técnicas de coleta de dados estão descritas no subitem 4.2.

\subsection{Formação de professores}

Uma vez que não havia registros de uso de redes sociais educativas nem de outras plataformas de EAD em nenhum curso de música da cidade, até à ocasião da pesquisa, o pesquisador propôs um curso de extensão pela Universidade Federal de Pernambuco 
(UFPE) intitulado "Tecnologias Digitais Aplicadas ao Ensino da Música", abordando não só o uso de redes sociais educativas, mas temas relacionados a outras tecnologias, facilitando, assim, o acesso ao campo de pesquisa.

Com uma carga horária de 24 horas/aula, distribuídas em 3 semanas consecutivas, o curso foi destinado aos professores de música que já atuam profissionalmente e aos graduandos ou pós-graduandos em Música. Participaram 21 interessados, incluindo professores de escolas privadas e da rede pública de ensino das 3 esferas de governo: municipal, estadual e federal. Os participantes do curso declararam ao final do curso que, embora não possuíssem experiência prévia com AVAs, consideraram positiva a experiência e que a Openredu, enquanto uma rede social educativa, é uma ferramenta válida para a aprendizagem de Música.

A partir de então, houve interesse de alguns concluintes do curso de extensão em utilizar a Openredu em suas práticas docentes, pelo que seria possível aplicar o experimento num total de 4 escolas de música: uma confessional e outra municipal que oferecem curso básico de música, uma escola estadual, de nível técnico, e a Escola de Música da UFPE, onde se deu o experimento. O referido curso serviu, portanto, como ponto de partida para acesso ao campo de pesquisa; deste modo, este artigo focará apenas na análise do engajamento dos estudantes da graduação em Música, como descrito a seguir.

\subsection{O experimento}

Os participantes do experimento foram alunos concluintes do curso de Licenciatura em Música da UFPE, matriculados na disciplina Prática do Ensino da Música 2. Com a maior carga horária do curso, a disciplina se divide em três etapas, sendo a primeira etapa com encontros presenciais, em sala de aula; a segunda etapa prática, com regência em alguma escola da educação básica; e, por fim, um período para a elaboração de um relatório final. O AVA foi utilizado exclusivamente na primeira etapa.

Antes da primeira aula mediada pela plataforma Openredu, o professor orientou os alunos a criarem uma conta, junto ao pesquisador. Esta iniciativa serviu para capturar as telas durante o cadastro, a fim de verificar a usabilidade da Openredu para duas atividades ou ações principais: criar uma conta e matricular-se na disciplina. Para tanto, o pesquisador disponibilizou um computador e forneceu um roteiro, pelo qual, exclusivamente, as ações foram executadas.

O questionário utilizado nesta pesquisa foi elaborado a partir do documento utilizado pelo Australian Council for Educational Research - ACER (Conselho Australiano para Pesquisa Educacional) para diagnosticar anualmente o engajamento discente no ensino superior e a qualidade do ensino superior na Austrália e na Nova Zelândia, desde 2007 [Coates 2009].

Já a observação se restringiu apenas ao monitoramento do ambiente virtual, com o fim de verificar a usabilidade da plataforma, a dinâmica do processo de aprendizagem do assunto observado e indícios de engajamento.

Por sua vez, a entrevista semiestruturada, que foi facultada a todos os participantes, foi realizada com 7 participantes do curso de Licenciatura em Música da Universidade Federal de Pernambuco, incluindo o professor da disciplina, visando 
captar o motivo da atividade investigada e o impacto da adoção da modalidade mista com redes sociais educativas, a partir da percepção dos discentes e do professor. $\mathrm{O}$ roteiro da entrevista incluiu os fatores que contribuem para a eficácia de um ambiente colaborativo, conforme Zurita e Nussbaum (2007).

A análise qualitativa dos dados foi realizada com o software NVivo, categorizando o perfil dos participantes quanto à habilidade com computadores, suas experiências com a modalidade mista ou mesmo com a modalidade a distância, suas expectativas sobre a modalidade mista e necessidades especiais. Quanto ao engajamento, a categorização foi sintetizada com a motivação para a realização da atividade, responsabilidade e apoio mútuo para aprender, sensação de pertencimento à comunidade escolar, mecanismos e recursos utilizados pelo professor e sugeridos pelos alunos, usabilidade para a aprendizagem, e fatores que impactam o engajamento, conforme seção seguinte.

\section{Análise do engajamento}

$\mathrm{Na}$ Openredu as aulas são organizadas assim: Ambiente $=>$ Curso $=>$ Disciplina $=>$ Módulo $=>$ Aula. A aula postada na plataforma Openredu, e que serviu como objeto de análise para esta pesquisa "Como se prepara uma aula?". A disciplina Prática do Ensino da Música 2 seria a única disciplina em todo o perfil do curso em que os licenciandos atuam como professores regentes.

A Teoria da Atividade embasou a diagramação da atividade proposta pelo professor, e permitiu a análise da efetividade da modalidade mista com o uso da Openredu. Antes de apresentar os fatores de impacto positivo e negativo sobre o engajamento discente na modalidade mista de ensino, convém destacar a importância da discussão trazida por Campello (2005) sobre a heurística memorability, de Nilsen (1993). Campello (2005) sugere que a usabilidade de uma plataforma de educação a distância seja avaliada no nível da díade atividade-ação, estando o nível operacional num plano de ação interno e inconsciente [Barbosa e Silva 2010]. Quando a ação se torna uma operação, há o que estes autores chamam de internalização. E é exatamente no processo de internalização que reside a usabilidade para a aprendizagem; inclusive, porque a ação humana é direcionada primeiramente a um objeto ou a outro indivíduo. Conclui-se, então, que problemas de usabilidade numa plataforma de EAD podem causar desengajamento nos usuários.

De modo geral os participantes desta pesquisa atestaram aceitação tanto para a modalidade mista quanto para a plataforma utilizada: "[...] de fato, foi uma experiência legal", afirmou um deles.

As escalas pautadas por Coates (2009) no Student Engagement Questionnaire SEQ, sugerem que a Instituição de Ensino é corresponsável pelo engajamento discente, desafiando-os a aprender de forma ativa, e ampliando suas experiências educacionais, e deve estimular uma interação interpessoal entre os membros da comunidade escolar, a fim de que se legitime o sentimento de pertencimento a essa comunidade. A interação dos alunos com seus pares, com o professor e com os artefatos pode contribuir para autonomia do indivíduo em situações não didáticas [Tauber 1999; Toczek 2004]. 
No início do experimento de nossa pesquisa, quando da aplicação do questionário, os alunos participantes se consideraram engajados no processo de aprendizagem, sendo a prática musical e a qualificação dos professores os melhores aspectos que o curso possuía para envolver os alunos. Vale ressaltar que para analisar o engajamento discente a partir da inserção da modalidade mista com o uso de uma rede social educativa é importante considerar a percepção do aluno quanto ao seu próprio engajamento no início do processo, cuja modalidade era presencial, até então. Contudo, os participantes sugerem que a Instituição deveria estimular principalmente maior interação entre alunos e professores, a fim de melhorar o engajamento discente. Esta prerrogativa, que perpassa por todas as modalidades de ensino, confirma os estudos de Palloff e Pratt (1999), e também de Fresen (2007), o qual atesta que o engajamento do aluno (enquanto aluno) prescinde da interação pessoal com outros alunos; de igual modo, remete principalmente às escalas de engajamento de Coates (2009). Além disso, através da fala dos alunos sobre mudanças na postura da Instituição para aumentar o engajamento, pôde-se abstrair um interesse em atividades grupais, e também sugestão de uso mais efetivo das tecnologias e maior integração da Instituição com o mercado de trabalho, aproximando-se do que Coates (2009) chamaria de Aprendizagem integrada ao trabalho.

Quanto às métricas de Green et al. (2008), as quais definem o sucesso escolar dos alunos a partir de três comportamentos, os participantes declararam o hábito de concluir as atividades propostas pelo professor e consideraram que a aula presencial é otimizada se antecipada ou complementada pelo ambiente virtual. O depoimento de um dos participantes confirmou que a adoção da modalidade mista, com uso de uma rede social educacional interferiu positivamente em seu engajamento escolar:

\begin{abstract}
Interferiria... [...] pra algo que é bom, porque eu me concentro melhor quando eu "tô" sozinho, consigo observar o conteúdo melhor, e... como eu lhe disse: se houvesse essa opção desde o início do curso, talvez a gente pudesse até aproveitar melhor algumas situações. Porque [...] não dá pra... pra... ver tudo dentro de um tempo limitado, que é dentro de uma sala de aula. Em casa, você procura o melhor horário, que você acredita estar relaxado, e aí você começa ver aquele conteúdo, e já chega em sala de aula [...] bem melhor. A aula fica mais dinâmica, a aula fica mais rápida, você pensa no que vai falar, em casa, e acaba falando em sala de aula, do que chegar aqui cru.
\end{abstract}

A compreensão da disciplina e das atividades a ela inerentes, bem como a importância do grupo sob a ótica do aluno são fatores que podem impactar o engajamento, como está implícito nos estudos de Green et al. (2008); Beer, Clarck e Jones (2010); Chen, Gonyea e Kuh (2008); Bulger et al. (2008); Zurita e Nussbaum (2007); Yonezawa, Jones e Joselowsky (2009); entre outros.

O discurso dos participantes revelou que a interação com os colegas, a atuação do professor e a própria modalidade mista são fatores que podem corroborar com o aumento do engajamento. Conforme relatado pelos alunos, as dificuldades iniciais relacionadas à usabilidade da Openredu foram superadas exatamente pela semelhança da Openredu com outras redes sociais das quais eles já participavam, como o facebook, por exemplo. Finalmente, destacaram-se como fatores que podem impactar 
negativamente o engajamento dos alunos a falta de interação síncrona e feedback, a didática do professor e fatores relacionados à Openredu, quais sejam: quantidade limitada de caracteres para comentários no mural e o modo como esses comentários são organizados, e a impossibilidade de fazer upload de arquivos.

\section{Considerações finais}

Os fatores que impactam o engajamento são as variáveis que apareceriam implicitamente no processo educativo. Através da análise qualitativa, foi possível perceber que a interação com os colegas, a atuação do professor e a modalidade mista por si só são fatores que podem contribuir para o engajamento do aluno. Por outro lado, a atuação do professor também pode interferir negativamente no engajamento discente, assim como a falta de interação síncrona e de feedback.

Segundo a Teoria da Atividade [Leontiev 1978], referencial que embasou a pesquisa, o fenômeno engajamento decorre exatamente das interações do sujeito com a comunidade, incluindo as interações presenciais (modalidade mista), sincronização (o caso das comunicações síncronas e assíncronas na Openredu), relações de coordenação e negociação (papéis do professor). Enfim, a aprendizagem ativa, as interações pessoais e o enriquecimento das experiências educacionais - componentes da escala de engajamento de Coates (2009) - foram pontuados pelos alunos em todo o experimento, confirmando a hipótese de que a modalidade mista com o uso de uma rede social educativa pode melhorar o engajamento do aluno, no contexto da aprendizagem musical.

\section{Referências}

Barbosa, S. D. J. e Silva, B. S. (2010) “Interação humano-computador", Elsevier, Rio de Janeiro.

Beer, C., Clark, K, Jones, D. (2010) "Indicators of engagement" In 27th Annual Ascilite Conference, New South Wales, Sydney.

Bertelsen, O. W. e Bødker, S. (2003) "Activity Theory", "HCI Models, Theories and Frameworks: toward a multidisciplinary science", Edited by Carroll, J. M., Morgan Kaufmann, San Francisco, CA.

Brasil. Lei n. 11.769, de 18 de agosto de 2008. Dispõe sobre a obrigatoriedade do ensino da música na educação básica. Brasília: 2008.

Bulger, M. E., Mayer, R. E.; Almeroth, K. C. e Blau, S. D. (2008) "Measuring Learner Engagement in Computer-Equipped College Classrooms" In Journal of Educational Multimedia and Hypermedia, pages 129-143. Chesapeake.

Campello, S. B. (2005) "Usability for Learning: A Socio-Cultural Approach to the Usability of VLEs", PhD thesis, The University Of Reading - UK.

Chen, P., Gonyea, R. e Kuh, G. (2008) "Learning at a Distance: Engaged or Not?" In Innovate: Journal of Online Education, 4(3).

Coates, H. B. (2009) "Engaging students for success: Australasian Student Engagement Report", http://www.acer.edu.au/files/AUSSE_Australasian-Student-EngagementReport-ASER-2008.pdf, January 2013. 
CBIE-LACLO 2015

Anais do XXI Workshop de Informática na Escola (WIE 2015)

Colabardini, J. C. M. e Oliveira, M. R. G. (2013) "A Educação Musical a Distância nas Páginas da ABEM", In 5th Seminário Internacional de Educação a Distância, pages 384-395, Minas Gerais.

Fresen, J. (2007) "A Taxonomy of Factors to Promote Quality Web-Supported Learning" In International Journal on E-Learning, 6 (3), Pages 351-362, Chesapeake.

Gohn, D. (2011) "Educação Musical a Distância: Abordagens e Experiências", Cortez, São Paulo.

Gomes, A. S. et al. (2012) "Educar com o Redu", Redu Educational Technology, Recife.

Green, G., Rhodes, J., Hirsch, A. H., Suarez-Orozco, C. e CAMIC, P. M. "Supportive Adult Relationships and the Academic Engagement of Latin American Immigrant Youth" In Journal of School Psychology, 46, pages 393-412.

Henderson Filho, J. R. (2007) "Formação Continuada de Professores de Música em Ambiente de Ensino e Aprendizagem Online”, Tese de Doutorado, UFRGS, Porto Alegre.

Lakatos, E. M., Marconi, M. A. (1991) "Fundamentos de metodologia científica”, Atlas, São Paulo.

Lim, C. P. e Hang, D. (2003) "An Activity Theory Approach to Research ICT Integration in Singapore Schools" In Computers \& Education, 41(1), pages 49-63.

Kuh, G. D. (2001) "Assessing What Really Matters to Student Learning. Inside the National Survey of Student Engagement", http://cpr.indiana.edu/, July 2015.

Malheiros, B. T. (2011) "Metodologia da Pesquisa em Educação”, LTC, Rio de Janeiro.

Martins, L. E. G. (2007) "Teoria da Atividade: Um Paradigma Possível para Elicitação de Requisitos de Software” In 3rd WOSES, Pernambuco.

McGrath, G. M. e Uden, L. (2000) "Aspects of the Software Development Process: An Activity Theory Based Approach" In 33th Hawaii International Conference on System Sciences, USA.

Morais, C., Miranda, L., Alves, P., Dias, P. (2011) “Actividades Desenvolvidas nas Redes Sociais por Estudantes do Ensino Superior" In 7rd Conferência Internacional de TIC na Educação, Braga, Portugal.

Nilsen, J. (1993) “Usability Engineering”, Academic Press, New York.

Oliveira, F. K. e Oliveira, O. S. (2012) "Edmodo: Uma Rede Social Educacional" In 4th Simpósio Hipertexto e Tecnologias na Educação, Pipa Comunicação, Recife.

Oliveira-Torres, F. A. (2012) "Pedagogia Musical Online: Um Estudo de Caso no Ensino Superior de Música a Distância”, Tese de Doutorado, UFRGS, Porto Alegre.

Palloff, R. M. e Pratt, K. (1999) "Building learning communities in cyberspace”, JosseyBass, San Francisco. 
Rodrigues, C. S. et al. "A Peculiaridade do Ambiente Virtual de Aprendizagem na Educação a Distância: Uma Análise do Ambiente Moodle" In 9th Congresso Internacional de Tecnologia na Educação, Pernambuco.

Rosa, I. R. (2012) "Comunicação dos Jovens nas Redes Sociais da Internet" In 4th Simpósio Hipertexto e Tecnologias na Educação, Pipa Comunicação, Recife.

Schneider, E. I., Suhr, I. R. F., Rolon, V. E. K. e Almeida, C. M. (2013) "Sala de Aula Invertida em EAD: Uma Proposta de Blended Learning" In Revista Intersaberes, $8(16)$, pages $68-81$.

Sheridan, R. (2009) "Hybrid and Blended Learning Approaches for Internet-Based Online Education" In Wilberforce University Faculty Journal, http://www.academia.edu/4112227/Hybrid_and_Blended_Learning_Approaches_for _Internet-Based_Online_Education, August 2013.

Soares, A. P. O. (2012) “A Interação em Fóruns de Discussão na EAD e Fóruns de Comunidades do Orkut: Um Estudo Comparativo do Gênero" In 4th Simpósio Hipertexto e Tecnologias na Educação, Pipa Comunicação, Recife.

Sylvestre, A. P. M. (2012) "Redes Sociais, Poder, Ensino" In 4th Simpósio Hipertexto e Tecnologias na Educação, Pipa Comunicação, Recife.

Tang, Y. (2010) "Teaching Application Study of Hybrid Learning Model" In International Conference on E-Health Networking, Digital Ecosystems and Technologies, Shanghai.

Tauber, T. R. (1999) "Classroom management, sound theory and effective practice", Bergin \& Garvey, Westport.

Toczek, M. C. e Martinot, D. (2004) "Le Défi Éducatif: Des Situations pour Réussir", Armand Colin, Paris.

Valdez, M.T., Ferreira, C.M., Barbosa, F.P.M. (2013) "Desktop VR Systems - A Distance Learning Method and Technology" In 4th IEEE Global Engineering Education Conference, Berlin.

Vygotsky, L.S. (1978) "Mind in Society: The Development of Higher Psychological Processes", Edited by M. Cole, V. John-Steiner e S. Scribner, Havard University Press, Havard.

Werstch, J. V. (1981) "The Concept of Activity in Soviet Psychology", M. E. Sharp, Minnesota.

Yang, H., Tang, J. (2003) "Effects of Social Network on Student's Performance: A Web-Based Forum Study in Taiwan" In JALN, 7(3).

Yonezawa, S., Jones, M. e Joselowsky, F. (2009) "Youth Engagement in High Schools: Developing a Multidimensional, Critical Approach to Improving Engagement for All Students" In J Educ Change, https://escholarship.org/uc/item/9425g258\#page-1.

Zurita, G. e Nussbaum, M. (2007) "A Conceptual Framework Based on Activity Theory for Mobile CSCL" In British Journal of Educational Technology, 38(2), pages 211235. 